\title{
Effect of postmastectomy radiotherapy on triple-negative breast cancer with T1-2 and 1-3 positive axillary lymph nodes: a population-based study using the SEER 18 database
}

\author{
Jie Zhang ${ }^{1, *}$, Xiao-Xiao Wang ${ }^{2, *}$, Jun-Yu Lian ${ }^{2, *}$ and Chuan-Gui Song ${ }^{1}$ \\ ${ }^{1}$ Department of Breast Surgery, Affiliated Union Hospital, Fujian Medical University, Fuzhou, China \\ ${ }^{2}$ Department of Gynecology and Obstetrics, Fujian Provincial Maternity and Children Health Hospital, Teaching Hospital of \\ Fujian Medical University, Fuzhou, China \\ *These authors have contributed equally to this work \\ Correspondence to: Chuan-Gui Song, email: songchuangui@yahoo.com \\ Keywords: postmastectomy radiotherapy; triple-negative breast cancer; 1-3 positive lymph nodes; overall survival; breast cancer- \\ specific survival \\ Received: October 31, $2017 \quad$ Accepted: February 24, $2018 \quad$ Published: August 27, 2019 \\ Copyright: Zhang et al. This is an open-access article distributed under the terms of the Creative Commons Attribution License 3.0 \\ (CC BY 3.0), which permits unrestricted use, distribution, and reproduction in any medium, provided the original author and source \\ are credited.
}

\section{ABSTRACT}

There is consensus on the routine use of postmastectomy radiotherapy (PMRT) in patients with four or more positive axillary lymph nodes. However, the benefits of PMRT in patients with T1-2 and 1-3 involved lymph nodes still remain controversial. Data from the Surveillance, Epidemiology, and End Results Program (SEER) of the United States between 2010 and 2012 were used to analyze the outcomes of 675 triple-negative breast cancer (TNBC) patients with T1-2 and 1-3 lymph nodes involved. Those patients were subdivided into radiotherapy (RT) (312) and no-RT groups (363). After a median follow-up time of 37 months, Kaplan-Meier analysis showed that PMRT significantly improved overall survival (OS) but not breast cancerspecific survival (BCSS) in the total cohort of 675 patients $(P=0.033$ and $P=0.063)$. And it was demonstrated that PMRT were independently associated with increased OS according to univariate and multivariate analyses. However, no significant differences in BCSS or OS were observed between the groups stratified by the number of positive lymph nodes. In conclusion, PMRT significantly improved OS for TNBC patients with T1-2 and 1-3 lymph nodes involved. Additional prospective studies are needed to provide a stronger evidence base for choosing patients for PMRT.

\section{INTRODUCTION}

Breast cancer is a highly heterogeneous disease, which is reflected in different clinical, pathologic, and molecular features. Currently, breast cancers are classified into four molecular subtypes including, luminal A, luminal B, human epidermal growth factor receptor 2 (HER2) overexpressed, and triple-negative breast cancer (TNBC) subtypes according to estrogen receptor (ER), progesterone receptor (PR), and HER2 status by gene expression profiling $[1,2]$. The TNBC tumors are defined not having ER, PR, and HER2 expression, and it has a worse prognosis because of its aggressively malignant features compared to the luminal subtypes. Regional recurrence and distance metastasis, especially to visceral organs and soft tissues, occur more frequently in TNBC. In addition, TNBC patients confront a high rate of early recurrence within the first three years and higher mortality within the first five years compared with other subtypes $[3,4]$.

Adjuvant radiotherapy plays a pivotal role in the treatment of breast cancer, since it can reduce the risk of loco-regional recurrence and overall mortality $[5,6]$. And postmastectomy radiotherapy (PMRT) is considered a standard treatment for high-risk breast cancers patients 
with involvement of more than four positive axillary lymph nodes [7, 8]. However, it still remains controversial for patients with 1-3 lymph nodes to receive radiotherapy (RT). Treatment with PMRT is recommended by the National Comprehensive Cancer Network's clinical practice guidelines for patients with early-stage breast cancer with 1-3 positive axillary lymph nodes [9]. In the St. Gallen Breast Cancer Meeting in 2017, 54.5\% of experts supported PMRT being used as standard treatment for patients with $\mathrm{N}+1$ to 3 all patients. A subgroup analysis by the Danish Breast Cancer Cooperative Group (DBCG) $82 \mathrm{~B} \& \mathrm{C}$ randomized trial indicated that the survival benefit after PMRT was substantial and similar in patients with 1-3 and four or more positive lymph nodes [10]. A meta-analysis of 22 randomized trials from Early Breast Cancer Trial list's Collaboration Group (EBCTCG) in 2014 revealed that PMRT could reduce 10-year locoregional recurrence and 20-year breast cancer mortality for patients with one to three positive axillary lymph nodes [6]. However, the retrospective multicenter analysis (KROG 1418) reported that PMRT had no significant impact on loco-regional recurrence-free survival (LRRFS), disease-free survival (DFS), and overall survival (OS) in pT1-2N1 patients treated with taxane-based chemotherapy [11]. Collectively, no information about breast cancer molecular subtypes was presented in these studies.

Given the poor prognosis and aggressive characteristics of TNBC patients and the controversy surrounding PMRT as appropriate for patients with small tumors with 1-3 positive nodes, our study is designed to investigate the effect of postmastectomy radiotherapy on OS and breast cancer-specific survival (BCSS) among TNBC patients with T1-2 and 1-3 positive lymph nodes by utilizing population-based SEER data, and to further determine which patients are most likely to benefit from PMRT.

\section{RESULTS}

\section{Demographic and clinical characteristics of the study population}

A summary of demographic and clinical characteristics is shown in Table 1. A total of 675 patients met the eligibility criteria for our study, and all patients had undergone mastectomy. Among these patients, $53.8 \%(n=363)$ of the patients were classified as the no radiotherapy-received group (no-RT group), and 46.2\% $(\mathrm{n}=312)$ as the radiotherapy-received group (RT group). The median follow-up time was 37 months. The median number of examined lymph nodes in our study was 13 for both groups. Compared with the patients who received RT, the no-RT group presented a significantly higher proportion of one positive lymph node (64.1\% VS. 45.5\%; $\mathrm{P}<0.001)$. And $96.2 \%$ of the patients in RT group were offered chemotherapy while it was recorded that $78.8 \%$ of the no-RT group were offered chemotherapy. In addition, the other tumor characteristics, including age, race, marital status, laterality, histological grade and stage $\mathrm{T}$ status were similar between the two groups.

\section{Survival comparisons according to lymph node status and radiation status}

We used the Kaplan-Meier plot and log-rank tests to compare BCSS and OS between different subgroups according to lymph node their status and the results are illustrated in Figure 1. In the total cohort of 675 patients, significantly improved OS after PMRT was found, whereas BCSS was similar between the RT and no-RT groups $(P=0.033$ and $P=0.063)$. In addition, no significant differences in OS was observed between the groups stratified by the number of positive lymph nodes; $P=0.073, P=0.455, P=0.144$ for $1 \mathrm{LN}, 2 \mathrm{LNs}$, and $3 \mathrm{LNs}$. there was no significant difference in BCSS when patients were stratified by the number of $\mathrm{LN} ; P=0.181, P=0.452$, and $P=0.176$ for $1 \mathrm{LN}, 2 \mathrm{LNs}$ and 3 LNs.

\section{Analyses of prognostic factors using Cox proportional hazard regression models}

In order to further investigate the prognostic factors of patients with TNBC, BCSS and OS analyses via univariate and multivariate Cox proportional hazard regression models were performed and the results are presented in Supplementary Table 1 and Table 2, respectively. With univariate analyses, no married status was significantly associated with reduced OS and BCSS; hazard ratio (HR), 1.159 with a $95 \% \mathrm{CI}, 1.117$ to 2.174 and $P=0.009$ for OS and HR, 1.484 with a $95 \% \mathrm{CI}, 1.035$ to 2.127 and $P=0.032$ for BCSS. Compared with tumors sized $2 \mathrm{~cm}$ to $5 \mathrm{~cm}$ (T2), patients with $\leq 2 \mathrm{~cm}$ (T1) tumors had a better OS and BCSS with univariate analyses; HR, 0.617 with a $95 \% \mathrm{CI}, 0.416$ to 0.917 and $P=0.017$ for OS and HR, 0.548 with a $95 \% \mathrm{CI}, 0.353$ to 0.852 and $P=0.008$ for BCSS. This result retained significance in multivariate analyses of OS and BCSS (Table 2). In addition, PMRT was independently associated with increased OS according to univariate and multivariate analyses.

\section{Stratification analysis with the number of positive lymph nodes}

In order to further investigate the effect of PMRT for TNBC patients, we performed multivariate analysis, stratifying according to the number of positive lymph node, which ruled out the impact of unbalanced distribution of chemotherapy between RT and no-RT groups. As shown in Table 3, when HRs of the three subgroups were conducted, no significant improvements in BCSS and OS were observed after PMRT. 
Table 1: Demographics and clinical characteristics of the study population

\begin{tabular}{|c|c|c|c|c|c|}
\hline \multirow{2}{*}{ Characteristics } & \multicolumn{2}{|c|}{ No Radiotherapy $(n=363)$} & \multicolumn{2}{|c|}{ Radiotherapy (n=312) } & \multirow{2}{*}{$\mathbf{P}^{\mathbf{c}}$} \\
\hline & No. & $\%$ & No. & $\%$ & \\
\hline Median follow-up (month) (IQR) & \multicolumn{2}{|c|}{$37(27-48)$} & \multicolumn{2}{|c|}{$37(28-47)$} & \\
\hline Age (years) & & & & & 0.070 \\
\hline Median & \multicolumn{2}{|c|}{53} & \multicolumn{2}{|c|}{50} & \\
\hline$<\mathbf{5 0}$ & 147 & 40.5 & 148 & 47.4 & \\
\hline$\geq \mathbf{5 0}$ & 216 & 59.5 & 164 & 52.6 & \\
\hline Race & & & & & 0.225 \\
\hline White & 275 & 75.8 & 219 & 70.2 & \\
\hline Black & 64 & 17.6 & 64 & 20.5 & \\
\hline Other $^{\mathrm{a}}$ & 24 & 6.6 & 29 & 9.3 & \\
\hline Marital status & & & & & 0.367 \\
\hline Married & 231 & 63.6 & 188 & 60.3 & \\
\hline Not married ${ }^{b}$ & 132 & 36,4 & 124 & 39.7 & \\
\hline Laterality & & & & & 0.413 \\
\hline Left & 186 & 51.2 & 150 & 48.1 & \\
\hline Right & 177 & 46.8 & 162 & 51.9 & \\
\hline Histological Grade & & & & & 0.386 \\
\hline I & 1 & 0.3 & 0 & 0.00 & \\
\hline II & 45 & 12,4 & 31 & 9.9 & \\
\hline III $^{\mathbf{c}}$ & 317 & 87.3 & 281 & 90.1 & \\
\hline Stage $T$ & & & & & 0.468 \\
\hline T1 & 120 & 33.1 & 95 & 30.4 & \\
\hline $\mathbf{T 2}$ & 243 & 66.9 & 217 & 69.6 & \\
\hline Number of lymph node & & & & & $<0.001$ \\
\hline 1 & 223 & 61.4 & 142 & 45.5 & \\
\hline 2 & 103 & 28.4 & 92 & 29.5 & \\
\hline 3 & 37 & 10.2 & 78 & 25.0 & \\
\hline Chemotherapy & & & & & $<0.001$ \\
\hline Yes & 286 & 78.8 & 300 & 96.2 & \\
\hline No & 77 & 21.2 & 12 & 3.8 & \\
\hline
\end{tabular}

Abbreviation: IQR, interquartile range.

a Other includes American Indian/Alaskan native, and Asian/Pacific Islander.

${ }^{\mathrm{b}}$ Not married includes divorced, separated, single (never married), unmarried or domestic partner and widowed.

${ }^{\mathrm{c}} \mathrm{P}$ value was calculated among all groups with a Chi-squared test, and a bold type indicates significance. 


\section{DISCUSSION}

In the current study, we tried to investigate the effect of PMRT on BCSS and OS among TNBC patients with T1-2 and 1-3 positive lymph nodes utilizing populationbased SEER data. We found an improved OS after PMRT in T1-2 and 1-3 axillary lymph node positive patients. This is the first study to show the difference of OS in such patients, while adjuvant radiotherapy has been reported to contribute to local-regional disease control in previous studies.

Currently, PMRT is recommended for patients with one to three nodes according to international consensuses and guidelines. The evidence comes mainly from a metaanalysis in 2014 from EBCTCG, which suggested PMRT could reduce 10-year rates of locoregional recurrence (LRR) and 20-year rates of breast cancer mortality [6]. But the treatment is still controversial because the trials from EBCTCG were predominantly performed in the 1970s and 1980s. In that period chemotherapy regime commonly was cyclophosphamide, methotrexate, and 5 -fluorouracil (CMF) based, which is clearly inadequate for TNBC patients. The results do not reflect the advances in systemic therapy, as it has been confirmed that the taxane/anthracycline based chemotherapy regime could yield more significant benefits on survival than the CMF regimen [12-14]. In addition, the numbers of positive and dissected lymph nodes were considered as significant predictors for LRR [15], whereas the median number of resected lymph nodes was fewer than 10 in EBCTCG trial. Hence, this meta-analysis may not represent the current clinical practice. In contrast, our study recruited TNBC patients diagnosed from 2010 to 2012, who mainly received taxane/anthracycline-based chemotherapy regimens and the median number of examined lymph nodes was 13 for both of our study groups. Eliminating the above confounding factors, our results further forcibly corroborated that PMRT has an advantageous effect on the OS outcome for TNBC patients with T1-2 and 1-3 positive lymph nodes.

In clinical practice, considering the delayed complications from radiation including cardiac toxicity, lymphedema, skin fibrosis, and so on, some surgeons and radiation oncologist do not recommend PMRT to patients with 1-3 positive axillary lymph nodes. But TNBC is a special subtype of breast cancer. Patients with TNBC are likely to suffer poorer survival and are prone to early locoregional recurrence and distant metastasis. Previous studies have demonstrated that different molecular subtypes had different response to PMRT. Wen et al. [16] found that the HER2-enriched and TNBC subtypes were associated with significantly higher 5-year LRR rates, lower 5-year LRRFS rates, and poorer 5-year BCSS rates in pT1-2N1M0 breast cancer patients who did not undergo PMRT, compared with the luminal A subtype. This means that PMRT is more important in treatment of the TNBC than it is for luminal subtypes. But the effect of PMRT on TNBC patients remains controversial. Kyndi et al. [17] found that no significant OS improvement after PMRT

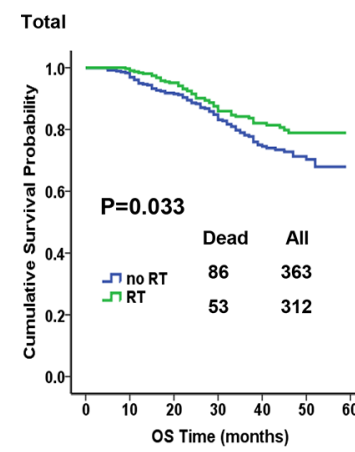

2

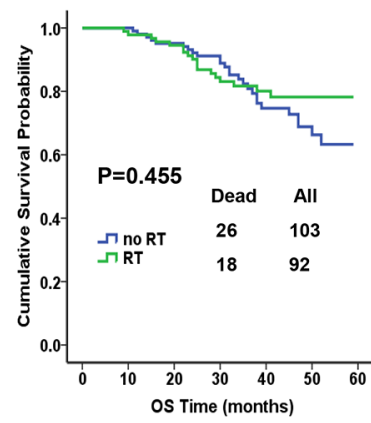

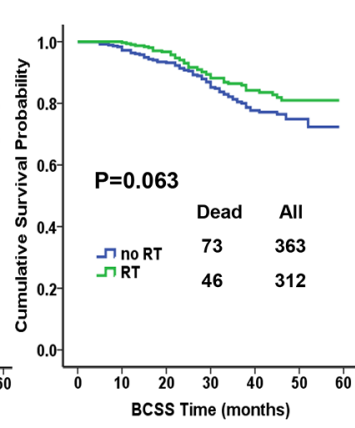

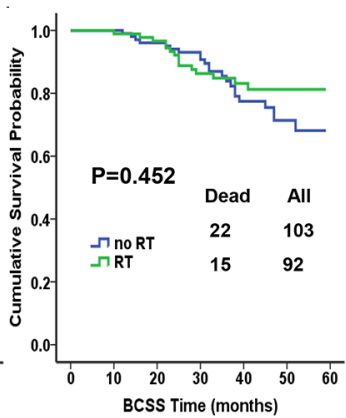

1
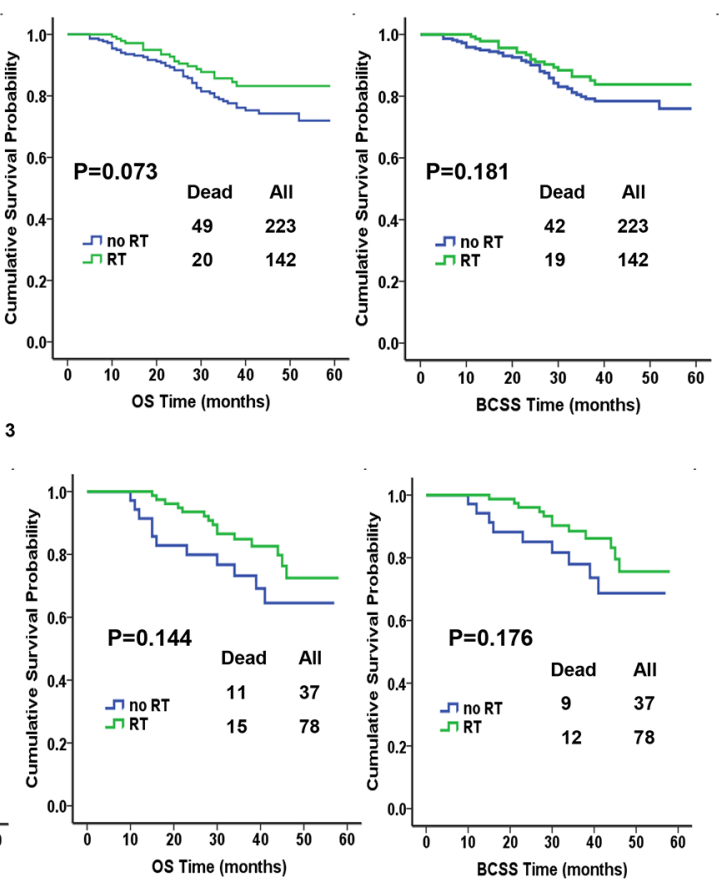

Figure 1: Kaplan-Meier plot and log-rank tests comparing overall survival (OS) and breast cancer-specific survival (BCSS) between RT and no-RT groups according to the number of positive lymph nodes. 
Table 2: Multivariate Cox proportional hazard model for the outcome-related factors

\begin{tabular}{|c|c|c|c|c|c|c|}
\hline \multirow{2}{*}{ Variable } & \multicolumn{3}{|c|}{ OS } & \multicolumn{3}{|c|}{ BCSS } \\
\hline & HR & $95 \% \mathrm{CI}$ & $\mathbf{P}^{\mathbf{a}}$ & HR & $95 \% \mathrm{CI}$ & $\mathbf{P}^{\mathbf{a}}$ \\
\hline \multicolumn{7}{|c|}{ Age (years) } \\
\hline$\geq \mathbf{5 0}$ & 1 & & & 1 & & \\
\hline$<\mathbf{5 0}$ & 0.841 & $0.598-1.183$ & 0.320 & 0.827 & $0.572-1.197$ & 0.314 \\
\hline \multicolumn{7}{|l|}{ Grade } \\
\hline III & 1 & & & 1 & & \\
\hline I/II & 1.088 & $0.645-1.835$ & 0.753 & 1.111 & $0.635-1.945$ & 0.711 \\
\hline \multicolumn{7}{|l|}{ Stage $T$} \\
\hline $\mathbf{T} 2$ & 1 & & & 1 & & \\
\hline T1 & 0.607 & $0.408-0.902$ & 0.014 & 0.544 & $0.349-0.846$ & 0.007 \\
\hline \multicolumn{7}{|c|}{ Number of lymph node } \\
\hline 1 & 1 & & & 1 & & \\
\hline 2 & 1.121 & $0.768-1.638$ & 0.553 & 1.065 & $0.707-1.603$ & 0.764 \\
\hline 3 & 1.269 & $0.797-2.020$ & 0.316 & 1.143 & $0.685-1.905$ & 0.609 \\
\hline \multicolumn{7}{|c|}{ Chemotherapy } \\
\hline Yes & 1 & & & 1 & & \\
\hline No & 1.029 & $0.631-1.678$ & 0.908 & 0.822 & $0.464-1.457$ & 0.502 \\
\hline \multicolumn{7}{|c|}{ Radiotherapy } \\
\hline No & 1 & & & 1 & & \\
\hline Yes & 0.661 & $0.460-0.949$ & 0.025 & 0.664 & $0.450-0.979$ & 0.039 \\
\hline
\end{tabular}

Abbreviation: CI, confidence interval.

${ }^{a} \mathrm{P}$ value was adjusted by a multivariate Cox proportional hazard regression model including age, grade, stage $\mathrm{T}$, the number of positive lymph nodes, chemotherapy and radiotherapy, and a bold type indicates significance.

was observed among patients with hormone receptor negative tumors. The authors speculated that this was due to the highly proliferative, aggressive behavior and radioresistance of TNBC subtype. Conversely, Chen et al. [18] confirmed the beneficial impact of PMRT on the DFS outcomes in patients with TNBC with intermediaterisk disease (stage $\mathrm{T} 1-\mathrm{T} 2 \mathrm{~N} 1$ ), which is consistent with our results. Accordingly, since TNBC is an aggressive disease and those patients can benefit from PMRT, why not strongly recommend them to receive PMRT to prolong survival? When making the decision to undergo PMRT or not, we should balance the benefits vs. toxicity and the tumor phenotype should be taken into consideration.

Although no statistical difference was observed for BCSS among the total cohort and by stratified analysis, a benefit trend was reflected from Kaplan-Meier analysis which was consistent with the trend in OS, thus these observations should be interpreted with caution. We speculated that the small percentage of patients who have died from breast cancer and the small sample size of patients who died in subgroup analysis could be one potential explanation for the finite benefits from PMRT. And hopefully will extending the follow-up time make our results more satisfactory.

There are several limitations of our study. Firstly, we were compelled to focus on short follow-up time because the information regarding HER-2 expression in the SEER database was not available until 2010. But for the TNBC subtype, an early peak of recurrence occurs within the first 2-3 years after diagnosis. Secondly, the SEER database is absent from information about LRR. However, information about chemotherapy is offered by the latest version of the SEER database. And we endeavored to mitigate several confounding variables, such as endocrine and trastuzumab treatment by selecting TNBC as the object of our study rather than other subtypes.

In conclusion, our study shares some similar results with the recent literature that there is a significant 
Table 3: Multivariate Cox proportional hazard model assessing the effect of postmastectomy radiotherapy stratified by the number of positive lymph node

\begin{tabular}{|c|c|c|c|c|c|c|}
\hline \multirow{2}{*}{$\begin{array}{l}\text { Number of positive } \\
\text { lymph node / Radiation }\end{array}$} & \multicolumn{3}{|c|}{ OS } & \multicolumn{3}{|c|}{ BCSS } \\
\hline & HR & $95 \% \mathrm{CI}$ & $\mathbf{P}^{\mathbf{a}}$ & HR & $95 \%$ CI & $\mathbf{P}^{\mathbf{a}}$ \\
\hline \multicolumn{7}{|l|}{ Total } \\
\hline RT & 0.661 & $0.460-0.949$ & 0.025 & 0.664 & $0.450-0.979$ & 0.039 \\
\hline no $R T$ & 1 & & & 1 & & \\
\hline \multicolumn{7}{|l|}{$1 \mathrm{LN}$} \\
\hline RT & 0.640 & $0.376-1.090$ & 0.100 & 0.674 & $0.388-1.171$ & 0.161 \\
\hline no RT & 1 & & & 1 & & \\
\hline \multicolumn{7}{|l|}{2 LNs } \\
\hline RT & 0.765 & $0.415-1.411$ & 0.391 & 0.741 & $0.381-1.441$ & 0.377 \\
\hline no RT & 1 & & & 1 & & \\
\hline \multicolumn{7}{|l|}{3 LNs } \\
\hline RT & 1 & & & 1 & & \\
\hline no RT & 1.841 & $0.795-4.267$ & 0.155 & 1.796 & $0.693-4.656$ & 0.228 \\
\hline
\end{tabular}

Abbreviation: CI, confidence interval.

${ }^{\text {a }} \mathrm{P}$ value was adjusted by a multivariate Cox proportional hazard regression model including age, grade, stage $\mathrm{T}$, chemotherapy and radiotherapy, and a bold type indicates significance.

improvement on OS after PMRT among TNBC patients with T1-2 and 1-3 positive axillary lymph nodes. In accordance with current consensuses and guidelines, we suggest that PMRT should be strongly considered in T1-2N1 breast cancer patients particularly in TNBC, an aggressive and poor prognosis disease. Additional prospective randomized studies are needed to provide a stronger evidence base for selecting patients for PMRT.

\section{MATERIALS AND METHODS}

\section{Ethics statement}

The data in the SEER database do not require informed patient consent because cancer is a disease reported by every state of the United States. But a DataUse Agreement for the SEER 1973-2014 Research Data File has been completed.

\section{Patients}

SEER*Stat version 8.3.4 was used to generate a case listing. We identified 675 patients screened according to the following inclusion criteria: female; year of diagnosis from 2010 to 2012; age of diagnosis between 20 years and 80 years; breast cancer as the first and only malignant cancer diagnosis; pathologically confirmed invasive ductal carcinoma-not otherwise specified (ICD-O-3 8500/3), unilateral cancer; TNBC subtype (absence of ER, PR, and HER2); histological grades I-III; AJCC T stagesI-II; known tumor size category; known lymph node status; record of chemotherapy therapy, mastectomy received. We excluded patients with inflammatory breast cancer, in situ disease, and no record of radiotherapy. We calculated follow-up durations from January 1, 2010 to December 31, 2014 to ensure adequate follow-up duration.

\section{Statistical analyses}

Patients were divided into RT group and no-RT group categorized by radiotherapy status. A Chi-squared test was used to compare the demographic and clinical characteristics of the included cases between the two groups. The Kaplan-Meier analysis was performed to generate survival curves, and the log-rank test was used to compare the unadjusted BCSS and OS rates of patients with different lymph node status. BCSS was measured from the date of diagnosis to the date of breast cancer death. OS was defined as the time from the date of diagnosis to the date of death due to any cause (including breast cancer) or the last follow-up. Adjusted hazard ratios (HRs) with 95\% confidence interval (CI) were calculated using a Cox proportional hazard regression model to estimate the outcome-related factors. All $P$-values were two-sided and $P<0.05$ were considered statistically significant. All statistical analyses were carried out 
using SPSS version 22.0 software (IBM SPSS Statistics, Chicago, IL, US).

\section{CONFLICTS OF INTEREST}

The authors have no conflicts of interest.

\section{FUNDING}

This study was funded by the National Natural Science Foundation of China (81672817) the Wu Jieping Medical Foundation (320.6750.14142).

\section{REFERENCES}

1. Cancer Genome Atlas Network. Comprehensive molecular portraits of human breast tumours. Nature. 2012; 490: 61-70. https://doi.org/10.1038/nature11412. [PubMed]

2. Perou CM, Sorlie T, Eisen MB, van de Rijn M, Jeffrey SS, Rees CA, Pollack JR, Ross DT, Johnsen H, Akslen LA, Fluge O, Pergamenschikov A, Williams C, et al. Molecular portraits of human breast tumours. Nature. 2000; 406: 74752. https://doi.org/10.1038/35021093. [PubMed]

3. Foulkes WD, Smith IE, Reis-Filho JS. Triple-negative breast cancer. N Engl J Med. 2010; 363: 1938-48. https:// doi.org/10.1056/NEJMra1001389. [PubMed]

4. Dent R, Trudeau M, Pritchard KI, Hanna WM, Kahn HK, Sawka CA, Lickley LA, Rawlinson E, Sun P, Narod SA. Triple-negative breast cancer: clinical features and patterns of recurrence. Clin Cancer Res. 2007; 13: 4429-34. https:// doi.org/10.1158/1078-0432.CCR-06-3045. [PubMed]

5. Clarke M, Collins R, Darby S, Davies C, Elphinstone P, Evans V, Godwin J, Gray R, Hicks C, James S, MacKinnon E, McGale P, McHugh T, et al. Effects of radiotherapy and of differences in the extent of surgery for early breast cancer on local recurrence and 15-year survival: an overview of the randomised trials. Lancet. 2005; 366: 2087-106. https://doi. org/10.1016/S0140-6736(05)67887-7. [PubMed]

6. McGale P, Taylor C, Correa C, Cutter D, Duane F, Ewertz M, Gray R, Mannu G, Peto R, Whelan T, Wang Y, Wang Z, Darby S, and EBCTCG (Early Breast Cancer Trialists' Collaborative Group). Effect of radiotherapy after mastectomy and axillary surgery on 10-year recurrence and 20-year breast cancer mortality: meta-analysis of individual patient data for 8135 women in 22 randomised trials. Lancet. 2014; 383: 2127-35. https://doi.org/10.1016/ S0140-6736(14)60488-8. [PubMed]

7. Frasier LL, Holden $\mathrm{S}$, Holden $\mathrm{T}$, Schumacher JR, Leverson G, Anderson B, Greenberg CC, Neuman HB. Temporal Trends in Postmastectomy Radiation Therapy and Breast Reconstruction Associated With Changes in National Comprehensive Cancer Network Guidelines. JAMA Oncol. 2016; 2: 95-101. https://doi.org/10.1001/ jamaoncol.2015.3717. [PubMed]
8. Tseng YD, Uno H, Hughes ME, Niland JC, Wong YN, Theriault R, Blitzblau RC, Moy B, Breslin T, Edge SB, Hassett MJ, Punglia RS. Biological Subtype Predicts Risk of Locoregional Recurrence After Mastectomy and Impact of Postmastectomy Radiation in a Large National Database. Int J Radiat Oncol Biol Phys. 2015; 93: 622-30. https://doi. org/10.1016/j.ijrobp.2015.07.006. [PubMed]

9. NCCN clinical practice guidelines in oncology: breast cancer, version 2 National Comprehensive Cancer Network. 2017.

10. Overgaard M, Nielsen HM, Overgaard J. Is the benefit of postmastectomy irradiation limited to patients with four or more positive nodes, as recommended in international consensus reports? A subgroup analysis of the DBCG 82 b\&c randomized trials. Radiother Oncol. 2007; 82: 247-53. https://doi.org/10.1016/j.radonc.2007.02.001. [PubMed]

11. Kim YJ, Park W, Ha B, Park B, Joo J, Kim TH, Park IH, Lee KS, Lee ES, Shin KH, Kim H, Yu JI, Choi DH, et al. Postmastectomy Radiotherapy in Patients with pT1-2N1 Breast Cancer Treated with Taxane-Based Chemotherapy: A Retrospective Multicenter Analysis (KROG 1418). Cancer Res Treat. 2017; 49:927-936. https://doi. org/10.4143/crt.2016.508. [PubMed]

12. Peto R, Davies C, Godwin J, Gray R, Pan H, Clarke M, Cutter D, Darby S, McGale P, Taylor C, Wang Y, Bergh J, Di Leo A, et al. Comparisons between different polychemotherapy regimens for early breast cancer: metaanalyses of long-term outcome among 100000 women in 123 randomised trials. The Lancet. 2012; 379: 432-44. https://doi.org/10.1016/s0140-6736(11)61625-5. [PubMed]

13. Sartor CI, Peterson BL, Woolf S, Fitzgerald TJ, Laurie F, Turrisi AJ, Bogart J, Henderson IC, Norton L. Effect of addition of adjuvant paclitaxel on radiotherapy delivery and locoregional control of node-positive breast cancer: cancer and leukemia group B 9344. J Clin Oncol. 2005; 23: 30-40. https://doi.org/10.1200/JCO.2005.12.044. [PubMed]

14. Mamounas EP, Bryant J, Lembersky B, Fehrenbacher L, Sedlacek SM, Fisher B, Wickerham DL, Yothers G, Soran A, Wolmark N. Paclitaxel after doxorubicin plus cyclophosphamide as adjuvant chemotherapy for nodepositive breast cancer: results from NSABP B-28. J Clin Oncol. 2005; 23: 3686-96. https://doi.org/10.1200/ JCO.2005.10.517. [PubMed]

15. Taghian A, Jeong JH, Mamounas E, Anderson S, Bryant J, Deutsch M, Wolmark N. Patterns of locoregional failure in patients with operable breast cancer treated by mastectomy and adjuvant chemotherapy with or without tamoxifen and without radiotherapy: results from five National Surgical Adjuvant Breast and Bowel Project randomized clinical trials. J Clin Oncol. 2004; 22: 4247-54. https://doi. org/10.1200/JCO.2004.01.042. [PubMed]

16. Wen G, Zhang JS, Zhang YJ, Zhu YJ, Huang XB, Guan XX. Predictive Value of Molecular Subtyping for Locoregional Recurrence in Early-Stage Breast Cancer with N1 without 
Postmastectomy Radiotherapy. J Breast Cancer. 2016; 19: 176-84. https://doi.org/10.4048/jbc.2016.19.2.176. [PubMed]

17. Kyndi M, Sorensen FB, Knudsen H, Overgaard M, Nielsen HM, Overgaard J, Danish Breast Cancer Cooperative Group. Estrogen receptor, progesterone receptor, HER-2, and response to postmastectomy radiotherapy in high-risk breast cancer: the Danish Breast Cancer Cooperative Group.
J Clin Oncol. 2008; 26: 1419-26. https://doi.org/10.1200/ JCO.2007.14.5565. [PubMed]

18. Chen X, Yu X, Chen J, Yang Z, Shao Z, Zhang Z, Guo $X$, Feng Y. Radiotherapy can improve the disease-free survival rate in triple-negative breast cancer patients with T1-T2 disease and one to three positive lymph nodes after mastectomy. Oncologist. 2013; 18: 141-7. https://doi. org/10.1634/theoncologist.2012-0233. [PubMed] 